\title{
Polylectrolyte- versus membrane-coated electrodes for energy production by Capmix salinity exchange methods
}

\author{
M.M. Fernández ${ }^{\mathrm{a}}$, R.M. Wagterveld ${ }^{\mathrm{b}}$, S. Ahualli ${ }^{\mathrm{a}}$, F. Liu ${ }^{\mathrm{b}}$, A.V. Delgado ${ }^{\mathrm{a}, *}$, \\ H.V.M. Hamelers ${ }^{\mathrm{b}}$ \\ ${ }^{a}$ Department of Applied Physics, School of Sciences, University of Granada, 18071, \\ Granada, Spain. \\ ${ }^{b}$ WETSUS, Centre of Excellence for Sustainable Water Technology, 8900 CC, Leeuwarden, \\ The Netherlands.
}

\begin{abstract}
In this paper we analyze the energy and power achievable by means of a recently proposed salinity gradient technique for energy production. The method, denominated soft electrode or SE, is based on the potential difference that can be generated between two porous electrodes coated with cationic and anionic polyelectrolytes. It is related to the Capacitive Donnan Potential (CDP) technique, where the electrical potential variations are mostly related to the Donnan potential, of ion-selective membranes in the case of CDP, and of the polyelectrolyte coating in SE. It is found that although SE is comparable to CDP in terms of energy production, it presents slower rates of voltage change, and lower achieved power. The separate analysis of the response of positively and negatively coated electrodes shows that the latter produces most of the voltage rise and also of the response delay. These results, together with electrokinetic techniques, give an idea on how the two types of polyelectrolytes adsorb on the carbon surface and affect differently the diffusion layer. It is possible to suggest that the SE technique is a promising one, and it may overcome the drawbacks associated to the use of membranes in CDP.

Keywords: Blue energy, capmix techniques, CDP, Donnan potential,
\end{abstract}

\footnotetext{
${ }^{*}$ Corresponding author

Email address: adelgado@ugr.es (A.V. Delgado)
}

Preprint submitted to Journal of Power Sources

September 30, 2015 
polyelectrolyte coating

\section{Introduction}

Natural mixing of water streams with different salinities is an abundant but largely unused power source. However, since the 1950's [1, it has been recognized as a clean renewable energy resource available worldwide. The research 5 to date tends to focus on the natural process of mixing river water with seawater in river mouths as the main source of salinity gradient (or blue) energy [2, 3, 4. Within the recent past, other salinity difference sources have also grown in importance. Concentrated brine water from desalination plants can be paired with waste water from a treatment facility, reducing desalination energy cost [5. The latest researches have also led to a new interest in low temperature $\left(<80^{\circ} \mathrm{C}\right)$ waste heat and waste water from the industrial sector to convert it into useful work. For instance, waste heat can be used to generate low and high concentration solutions for salinity difference energy either by membrane distillation process [6] or by using thermolytic solutions [7. In addition, small water temperature differences can be combined with salinity gradient for increased energy extraction by means of either thermal membrane properties [8] or changes in the capacitance of the electrical double layer [9. As an interesting alternative, it has been recently shown that mixing solutions with different dissolved $\mathrm{CO}_{2}$ concentrations is a related method which can be used to harvest energy from gas emissions [10, 11].

A number of techniques have been developed to obtain the Gibbs free energy that is released when two electrolyte solutions of different concentrations are mixed. The most advanced approaches are the membrane-based PRO (pressure retarded osmosis) [12, 13, 14 and RED (reverse electrodyalisis) [15, 16].

${ }_{25}$ They have different working principles, operating considerations and membrane properties 17, 18. PRO utilizes the osmotic pressure difference between two chambers to produce pressurized water to generate electricity through a hydroturbine. In other words, the flow of water molecules (but not ions) into a more 
concentrated solution is the driving force of the technique. RED, on the other hand, uses membranes for the transport of ions and not water molecules. PRO and RED have been demonstrated at pilot scale [19, 20] and are close to commercialization, although both still face some problems related to membrane performance and costs.

A recent approach, called capacitive mixing (capmix), comprises a group of techniques which are based on the variation of the potential difference between two porous electrodes by exchanging the ionic contents of the solution in contact with them [21, 22]. An important difference with respect to the previous technologies is that here the two solutions do not flow simultaneously, but they flow alternatively through the same compartment which is a cell made of two 40 oppositely faced activated carbon electrodes.

Two main techniques have been developed for the direct production of electrical energy based on this principle. In CDLE (capacitive double layer expansion method) the electrodes are charged with an external power supply in presence of a salty solution. When this is exchanged by fresh water, an increase of the cell potential is produced due to the associated decrease in the capacitance of the electrical double layer (EDL) 2, 23, 24, 25. Alternatively, in CDP (capacitive Donnan potential method) the voltage difference generation involves the use of ion selective membranes on each electrode, and the cell potential is controlled by the Donnan potential difference [3, 26]. Improvements of the method have led to recently reported increases in the extracted power achieved [27, 28]. In principle, the CDLE approach would be cheaper to produce and easier to implement due to absence of membranes, also associated to a lower internal resistance of the cell. However, problems with self-discharging have prevented a real advance of this technique [29, 30. In addition, physical, chemical and organic fouling inevitably associated to exposure of membranes to natural waters 31] might also be present in the bare carbon films used in CDLE, although systematic studies on this issue have not been performed yet.

In the current context, a very promising technique will come from combining CDP and CDLE methods. Recall that while CDLE needs a power supply to 
store ions in activated carbon electrodes, CDP works efficiently with ion selective membranes. Membranes in CDP play the role of the external voltage source in CDLE, generating by themselves a voltage difference between the electrodes. The core of the present approach is the spontaneous generation of charge on the electrodes without the use of either membranes or a power supply. It is just the physicochemical modification of the carbon particles that produces the required charge.

As previously shown by Ahualli et al. 32 , we can attribute the term soft electrodes to conductive electrodes made of an activated carbon core and a polyelectrolyte layer, either cationic or anionic. Hence the soft electrode (SE) method would allow the generation of electrical energy due to changes in both the EDL capacitance of the carbon electrodes and the Donnan potential of the polyelectrolyte layer when solutions are exchanged. It has been shown that such modification leads to an important reduction of the leakage current [29, 30]. Similarly to an auto-generated cycle [26], characteristic of the CDP method, there is no need for an external power supply, and electricity generation is directly produced by the mixing process. Like in the CDLE method, the potential difference in the cell is associated to the processes occurring on the particles themselves without the need of membranes. Hence a relatively thin polyelectrolyte layer suffices and likely results in a decrease of the overall cost of the method.

Although the possibilities of the SE technique as an efficient way of producing clean electrical energy from mixing solutions were demonstrated in a previous work [32], its results have never been compared to those achieved with CDP. However, such a comparison is of a great interest, as both methods share the mechanism of specific charging of each electrode by means of a film on the active carbon layer, and each of them might have its advantages and drawbacks. In addition, it was not explored before whether the characteristics (adsorbed amount, molecular weight, type of polyelectrolyte) of the charged polymer coating in SE might make some difference in the performace of the method. Hence, the purpose of this paper is to compare CDP and SE methods in order to find out whether the mentioned advantages of not needing membranes can be a chance 
to take the best of both capmix techniques but approaching CDP extracted power. With this aim, we will first analyze the differences between both methods related to potential rise in open circuit voltage (OCV) measurements, and extracted power, that is, the essential information of the capmix cycles. We will also analyze the working principles of soft electrodes for parameter optimization. This will be a first insight into polyelectrolyte layer behavior and is key for the improvement of the SE method.

\section{Materials and Methods}

We used the same laboratory scale cell for SE and CDP experiments [26].

It consists of two parallel graphite collectors coated with carbon films (Voltea B.V., The Netherlands) and facing each other. These two electrodes of $2 \times 2$ $\mathrm{cm}^{2}$ were separated $200 \mu \mathrm{m}$. Concentrated $\left(30 \mathrm{~g} \mathrm{~L}^{-1} \mathrm{NaCl}\right)$ and dilute $\left(1 \mathrm{~g} \mathrm{~L}^{-1}\right.$ $\mathrm{NaCl}$ ) saline solutions were alternately fed into the cell at a constant flow rate of $100 \mathrm{~mL} \mathrm{~min}{ }^{-1}$. Before the start of each experiment, the carbon electrodes were soaked in salt water.

Two ion exchange membranes from Fumatech (Germany) were used for CDP experiments: a Fumasep FAS anion exchange and a Fumasep FKS cation exchange, both of 30-40 $\mu \mathrm{m}$ thickness. For the SE method, soft conductive electrodes were prepared by contacting Voltea carbon films with respectively anionic and cationic polyelectrolyte solutions under magnetic stirring during $12 \mathrm{~h}$. After that time, the electrodes were placed on the cell as shown in Fig. 1. Two anionic polyelectrolytes were tested: poly (sodium 4-styrenesulfonate) or PSS, and poly(acrylic acid) or PAA. The cationic polymers investigated were PDADMAC (poly(diallyldimethyl ammonium chloride)) and PEI (poly(ethyleneimine)). All of them were purchased from Sigma Aldrich (USA) and the typical molecular weights $\left(\mathrm{M}_{w}\right)$ selected were: 70000 and $200000 \mathrm{~g} \mathrm{~mol}^{-1}$ for PSS; 100 000-200 000 and $200000-350000 \mathrm{~g} \mathrm{~mol}^{-1}$ for PDADMAC; $15000 \mathrm{~g} \mathrm{~mol}^{-1}$ for PAA and $2000 \mathrm{~g} \mathrm{~mol}^{-1}$ for PEI.

Additionally, an alternative SE procedure was also tested which started by 
dard PSS and PDADMAC solutions. We used Norit DLC Super 30 activated carbon particles (Norit Nederland B.V., The Netherlands). Afterwards, we dried out the particles and made the carbon slurry as described in [26]. This resulting slurry was cast on a graphite film.

125 CDP methods. OCV results were obtained by measuring the potential difference between the electrodes in open circuit when fresh and salty solutions were cyclically exchanged. Secondly, we performed constant current capmix cycles [26]. In these, the cell was connected in series with a galvanostat (IviumStat, water in open circuit, so that a potential difference was established between the electrodes. Next, we closed the circuit and applied a constant current, driving the potential to zero. The circuit was opened again and fresh water provoked a potential rise in the cell. Finally, the circuit was closed again and the curzero. The energy obtained is represented by the area of the cycles of potential difference versus transported charge cycles. In both kinds of experiments, the measurement starts by bathing the short-circuited electrodes in fresh water.

The electrophoretic mobility of the bare and treated carbon particles was in all cases measured by triplicate in a Malvern Nano ZS device (Malvern Instruments, UK), which works on the basis of the analysis of the phase shift of the light scattered by the particles due to the electrophoretic migration. Dynamic (or AC) electrophoresis was additionally used in the characterization of the polyelectrolyte coating of the particles. In fact, it has been shown 33 that this quantity is much more sensitive than standard electrophoresis to the structure of the charged interface. For our experiments, we used an Acoustosizer II from Colloidal Dynamics (USA), based on the electrokinetic sonic amplitude (ESA) method. In this, an AC electric field with frequency between 1 and 18 $\mathrm{MHz}$ is applied to the suspension, and from the amplitude and phase of the in150 
transmission microscopy (HRTEM) observations were performed in a FEI Titan G2 microscope (USA) equipped with an EDX analyzer.

\section{Results and Discussion}

Unless otherwise stated, the typical conditions of SE preparation were: 100 is an important drawback that should be addressed to improve the extracted power. Despite the different time responses of CDP and SE, a steady tendency towards constant voltage values is observed in both cases after the first solution exchange. This suggests that leakage can be considered negligible in the two

\subsection{Capmix cycles}

In this section, we describe the results obtained for SE and CDP methods in constant current capmix cycles. As our goal is comparing SE with an optimized 
CDP cycle in terms of energy and power, we will perform two kinds of SE cycles differing in cycling periods. Thus, Fig. 3 a shows the SE cycle obtained with the same timing as the optimum CDP cycle represented for comparison in Fig. 3b. With the aim of maximizing the energy obtained from SE method, even at reduced power, we also performed SE cycles extending the open circuit stages. This longer cycle is represented in Fig. 3r and it lasts as long as needed to reach the SE maximum voltage rise, i.e. approximately 4 times longer than the short cycle.

Fig. 4 shows the extracted energy and power of the cycles in Fig. 3 for different charging/discharging currents. It can be seen that the energy obtained by $\mathrm{SE}$ as compared to CDP is about $30 \%$ lower in the most favorable conditions. However, the main difference between the two approaches appears when the extracted power is considered. In this case the maximum extracted power for $\mathrm{SE}\left(50 \mathrm{~mW} \mathrm{~m}^{-2}\right)$ is about half that obtained from CDP $\left(105 \mathrm{~mW} \mathrm{~m}^{-2}\right)$. Although this is a promising result, it is necessary to further explore the SE process to improve it.

\subsection{Individual behavior of soft electrodes}

To tackle this time response issue that apparently prevents the SE method from becoming a competitive alternative to CDP, we analyze each side separately in $\mathrm{OCV}$ measurements. That is, we assembled the cell with non-treated carbon film on one side, and the coated (either the anionic PDADMAC or cationic PSS) electrode on the other side. Fig. 5 compares both measurements. Note how PSS generates a comparatively large voltage rise (AB) with slow time response, while the cationic polymer PDADMAC generates a smaller voltage rise $(\mathrm{AC})$ with a quick time response. These unexpectedly different electrode performances explain the above mentioned energy and power results (Fig. 4), and provides an interesting insight into the SE technique. In the following sections, we analyze which parameters can affect the described behaviors. 


\section{Polymer characteristics}

The effect of polymer characteristics such as molecular weight, concentration or structure on the SE performance will be analyzed in this section. Let us first consider the OCV measurements when the coating is PSS as compared to a different anionic polymer, PAA. The OCV results are plotted in Fig. 6a,b for different anionic polyelectrolye concentrations. As observed, an increase in concentration leads to a larger voltage rise in both cases. However, the concentration needed and the overall voltage rise is different for each polymer: higher potential elevation with lower concentration is found for PSS. Fig. 6a also demonstrates that changing the molecular weight from 200000 to 70000 has a negligible effect on OCV measurements. Focusing now on time response differences, we can conclude that the polyelectrolyte characteristics have no influence on the kinetics of the SE cycle for anionic polymers. For instance, for the highest concentration in each case, the time needed to reach $90 \%$ of maximum OCV is quite similar: $200 \mathrm{~s}$ for PSS and $240 \mathrm{~s}$ for PAA.

Similarly, Fig. 6e shows the results for the cationic polymer PDADMAC (attemps to use PEI were unsuccessful probably because this is a low conductivity polymer producing a high internal resistance of the electrode). Note that, as 225 in the case of PSS, although the voltage rise increases when increasing the concentration of PDADMAC, neither of the analyzed polymer characteristics has effect on the time response.

\section{Alternative SE fabrication procedure}

In an attempt to get an insight into how polyelectrolytes coat the electrodes, we tried an alternative SE fabrication procedure that consists of treating the carbon particles and afterwards forming the film, instead of coating already formed carbon films. This method allows us to separate the role of the individual particles from the effect of the graphite support. The characterization of the coated particles was carried out by means of electrophoretic mobility (both DC and $\mathrm{AC}$ ). As a reference, we also measured non-treated particles.

Fig. 7 represents the electrophoretic mobility of non-treated and polyelectrolyte- 
coated activated carbon particles as a function of $\mathrm{NaCl}$ concentration. As we can see, the particles were indeed coated by the cationic polyelectrolyte PDADMAC, as expressed by the positive mobility, while the same kind of measurements did not reveal any effect of anionic PSS on the mobility. Recall that the capmix experiments demonstrate that PSS is functional in producing voltage changes associated to ionic strength variations. Hence the results of Fig. $7 \mathrm{~A}$ can be explained by hypothesizing that PDADMAC effectively coats the carbon particles, whereas electrostatic repulsion provokes that the negative polyelectrolyte is unable to form such a homogeneous layer.

These arguments agree with dynamic mobility $\left(u_{d}\right)$ data, typically more sensitive to the nature of the polyelectrolyte coating. Fig. $7 \mathrm{~b}$ shows the effect of the field frequency on the modulus and phase of the mobility. The increase of the modulus of polyelectrolyte-coated particles compared to bare carbons is clear 250 in this figure, indicating that both kinds of coated particles are more charged (either positively or negatively, depending on the coating) than bare ones. The phase of the mobility is particularly illustrative of the differences between the particles: both bare and PSS-coated carbons show increasingly negative values (approaching the theoretical limit of $-90^{\circ}$ when the inertia would stop completely the motion). This tendency is slower for PSS-coated particles, precisely due to the larger charge provoking a larger surface conductivity. This is even more evident in the case of PDADMAC, whereby the decrease of the phase with frequency is in fact not observed (it can be expected at higher frequencies). This is a manifestation of a larger surface conductivity than in the case of either bare or PSS-coated particles 33. Summarizing, the electrokinetic data of Fig. 7 suggest that the two polyelectrolytes are linked differently to the particles.

One possible way to ascertain these widely differing adsorption mechanisms is to analyze the behavior of a simpler surface, such as pure graphite films (not covered with activated carbon, and hence with very low surface area). To that 265 aim, we simply immersed graphite collectors in PSS and PDADMAC solutions. Fig. 8 shows the OCV of a cell formed with each treated graphite film versus an untreated carbon electrode, compared to the results obtained with the whole 
carbon film (Fig. 5). Somewhat surprisingly, the results show that the activated carbon-free graphite film coated with PDADMAC behaves very similarly to the PDADMAC carbon coated electrode. On the contrary, the potential rise of the PSS-graphite film is much lower than in the case of the PSS-carbon electrodes. In order to elucidate the origin of the differences observed, we performed HRTEM+EDX analysis of the three kinds of carbon particles: although the electron microscope observations can hardly allow to distinguish the location clues. Fig. S1 (Supplementary Information) shows the carbon and chlorine distribution on the PDADMAC-treated particle: note the homogeneity of the $\mathrm{Cl}$ distribution, and its coincidence with that of carbon. We can conclude that the particles are uniformly coated with PDADMAC, as indicated by the presence of $\mathrm{Cl}$, the counterion of the charged groups. On the contrary, Fig. S2 demonstrates that PSS is adsorbed differently: the presence of $\mathrm{Na}$ and $\mathrm{S}$ is mainly limited to the pores of the outermost (thinnest) layers of the particle, and the adsorption is not as uniform.

This set of results jointly indicates that PSS coats the carbon film in a nonuniform way, probably reaching some depth in patches in the electrode. On the contrary, PDADMAC appears to produce a uniform layer, a configuration closer to a selective membrane, which reaches its Donnan potential in a very short time. In the case of PSS, ions encounter the polyelectrolyte as they diffuse in the activated carbon plug, and the potential rises as the concentration front reaches the adsorbed molecules. This explains the finding (not shown for brevity) that the time response (typically $200 \mathrm{~s}$ for steady OCV) of an electrode built with PSS-pretreated carbon particles is similar to that of carbon treated with the same polyelectrolyte (Fig. 5p. For the same reason, the response of PDADMACcoated electrodes to salinity changes can be observed even without the need of the activated carbon particles (Fig. 8). This also explains why the OCV of electrodes built with particles pretreated with PDADMAC is similar to that of untreated particles (data not shown). 


\section{Conclusions}

In this paper, we have compared the performances of two methods aimed

300 capmix technique or CDP (capacitive Donnal Potential) has reached so far the highest extracted power among capmix methods. However, some limitations still need to be considered, specially those concerning cost, fouling and the assembly difficulties. The technique proposed in this article is based on the simple deposit

of a polyelectrolyte layer on the carbon films, called Soft Electrode (SE) method. This new technique can become an alternative to CDP due to its easier electrode preparation.

The preliminary results suggest that the SE technique might become an efficient salinity gradient energy method. However, the main difference between the two approaches is the comparatively slow SE time response. In an attempt to clarify the reason for this drawback, we evaluated separately the two polyelectrolyte (anionic: PSS; cationic: PDADMAC) coated electrodes. We found that PSS-based coating leads to high open circuit voltage and slow response, whereas PDADMAC electrodes behave the opposite way (lower OCV and faster response). A possible explanation for this might be found in the following: PSS seems to adsorb inhomogeneously on the pores of the outermost carbon layers. Therefore both the voltage rise and time response depend on how the exchanging solutions reach the polyelectrolyte patches. On the contrary, in the case of PDADMAC, we probably have a homogeneous thin layer staying on the surface of the film, which reacts very quickly to salinity changes.

Soft electrodes appear to be a promising alternative or complement to existing capmix techniques. Further investigation on polymer selection, methods for film treatment and stability of the coating are needed to improve the power output. When time response issues have been solved, the SE technique can outperform other capmix techniques. 


\section{Acknowledgements}

The research leading to these results received funding from the European Union 7th Framework Programme (FP7/2007-2013) under agreement No. 256868. Further financial support from Ministerio de Economía y Competitividad of Spain (Project FIS2013-47666-C3-1-R) and Junta de Andalucía (Spain) project PE-2012-FQM0694 is also gratefully acknowledged.

\section{References}

[1] R. E. Pattle, Production of electric power by mixing fresh and salt water in the hydroelectric pile, Nature 174 (4431) (1954) 660-660.

[2] D. Brogioli, Extracting renewable energy from a salinity difference using a capacitor, Phys. Rev. Lett. 103 (5) (2009) 058501.

[3] B. B. Sales, M. Saakes, J. W. Post, C. J. N. Buisman, P. M. Biesheuvel, H. V. M. Hamelers, Direct power production from a water salinity difference in a membrane-modified supercapacitor flow cell, Environ. Sci. Technol. 44 (2010) 5661-5665.

[4] G. Z. Ramon, B. J. Feinberg, E. M. V. Hoek, Membrane-based production of salinity-gradient power, Energy Environ. Sci. 4 (2011) 4423-4434.

[5] A. Achilli, J. L. Prante, N. T. Hancock, E. B. Maxwell, A. E. Childress, Experimental results from ro-pro: A next generation system for low-energy desalination, Environ. Sci. Technol. 48 (2014) 6437-6443.

[6] S. Lin, N. Y. Yip, T. Y. Cath, C. O. Osuji, M. Elimelech, Hybrid pressure retarded osmosismembrane distillation system for power generation from low-grade heat: Thermodynamic analysis and energy efficiency, Environ. Sci. Technol. 48 (2014) 5306-5313.

350

[7] B. E. Logan, M. Elimelech, Membrane-based processes for sustainable power generation using wate, Nature 488 (2012) 313319. 
[8] B. B. Sales, O. S. Burheim, S. Porada, V. Presser, C. J. Buisman, H. V. Hamelers, Extraction of energy from small thermal differences near room temperature using capacitive membrane technology, Environ. Sci. Technol. Lett. 1 (9) (2014) 356-360.

[9] S. A. Ahualli, M. M. Fernández, G. R. Iglesias, Á. V. Delgado, M. L. Jimenez, Temperature effects on energy production by salinity exchange, Environ. Sci. Technol. 48 (2014) 12378-12385.

[10] H. Hamelers, O. Schaetzle, J. Paz-García, P. Biesheuvel, C. Buisman, Harvesting energy from co2 emissions, Environ. Sci. Technol. Lett. 1 (1) (2013) $31-35$.

[11] J. Paz-Garcia, O. Schaetzle, P. Biesheuvel, H. Hamelers, Energy from co2 using capacitive electrodes - a model for energy extraction cycles., J. Colloid Interface Sci. 418 (2014) 200-207.

[12] A. Seppala, M. J. Lampinen, Thermodynamic optimizing of pressureretarded osmosis power generation systems, J. Membr. Sci. 161 (1-2) (1999) $115-138$.

[13] T. Thorsen, T. Holt, The potential for power production from salinity gradients by pressure retarded osmosis, J. Membr. Sci. 335 (1-2) (2009) 103-110.

[14] T.-S. Chung, X. Li, R. C. Ong, Q. Ge, H. Wang, G. Han, Emerging forward osmosis (fo) technologies and challenges ahead for clean water and clean energy applications, Curr. Opin. Chem. Eng. 1 (3) (2012) 246 - 257.

[15] P. Dugocki, A. Gambier, K. Nijmeijer, M. Wessling, Practical potential of reverse electrodialysis as process for sustainable energy generation, Environ. Sci. Technol. 43 (17) (2009) 6888-6894, pMID: 19764265.

[16] J. Veerman, M. Saakes, S. J. Metz, G. J. Harmsen, Reverse electrodialysis: A validated process model for design and optimization, Chem. Eng. J. 166 (1) (2011) 256-268. 
[17] J. W. Post, J. Veerman, H. V. M. Hamelers, G. J. W. Euverink, S. J. Metz, K. Nymeijer, C. J. N. Buisman, Salinity-gradient power: Evaluation of pressure-retarded osmosis and reverse electrodialysis, J. Membr. Sci. $288(1-2)(2007) 218-230$.

[18] N. Y. Yip, M. Elimelech, Comparison of energy efficiency and power den-

[24] R. A. Rica, D. Brogioli, R. Ziano, D. Salerno, F. Mantegazza, Ions transport and adsorption mechanisms in porous electrodes during capacitive-mixing double layer expansion (cdle), J. Phys. Chem. C 116 (32) (2012) 1693416938. 
[25] M. Jiménez, M. Fernández, S. Ahualli, G. Iglesias, A. Delgado, Predictions of the maximum energy extracted from salinity exchange inside porous electrodes, J. Colloid Interface Sci. 402 (2013) 340 - 349.

[26] F. Liu, O. Schaetzle, B. B. Sales, M. Saakes, C. J. N. Buisman, H. V. M. Hamelers, Effect of additional charging and current density on the performance of capacitive energy extraction based on donnan potential, Environ. Sci. Technol. 5 (9) (2012) 8642-8650.

[27] B. B. Sales, O. S. Burheim, F. Liu, O. Schaetzle, C. J. N. Buisman, H. V. M. Hamelers, Impact of wire geometry in energy extraction from salinity differences using capacitive technology, Environ. Sci. Technol. 46 (21) (2012) 12203-12208.

[28] O. S. Burheim, F. Liu, B. B. Sales, O. Schaetzle, C. J. N. Buisman, H. V. M. Hamelers, Faster time response by the use of wire electrodes in capacitive salinity gradient energy systems, J. Phys. Chem. C 116 (36) (2012) 1920319210.

[29] M. Marino, L. Misuri, M. Jimnez, S. Ahualli, O. Kozynchenko, S. Tennison, M. Bryjak, D. Brogioli, Modification of the surface of activated carbon electrodes for capacitive mixing energy extraction from salinity differences, J. Colloid Interface Sci. 436 (0) (2014) 146 - 153.

[30] D. Brogioli, R. Ziano, R. A. Rica, D. Salerno, O. Kozynchenko, H. V. M. Hamelers, F. Mantegazza, Exploiting the spontaneous potential of the electrodes used in the capacitive mixing technique for the extraction of energy from salinity difference, Energy Environ. Sci. 5 (2012) 9870-9880.

[31] B. Mi, M. Elimelech, Chemical and physical aspects of organic fouling of forward osmosis membranes, J. Membr. Sci. 320 (12) (2008) 292 - 302.

[32] S. Ahualli, M. Jiménez, M. M. Fernández, G. Iglesias, D. Brogioli, Á. V. Delgado, Polyelectrolyte-coated carbons used in the generation of blue en- 

25241-25246.

[33] S. Ahualli, M. L. Jimnez, F. Carrique, A. V. Delgado, Ac electrokinetics of concentrated suspensions of soft particles, Langmuir 25 (4) (2009) 19861997.

\section{Figure captions}

Figure 1. Sketch of the capmix cell based on soft electrodes.

Figure 2. Open circuit measurements for soft (a) and membrane-coated (b) electrodes when we alternately introduce salt and fresh water in the working cell. Dark-shaded areas: $30 \mathrm{~g} \mathrm{~L}^{-1} \mathrm{NaCl}$; light-shaded: $1 \mathrm{~g} \mathrm{~L}^{-1}$.

Figure 3. Constant current cycles for SE (a and c) and membranes-coated electrodes (b). In a) and b) the periods are equal, while in c) the period of the SE cycle is approximately 4 times longer. The corresponding potential-charge cycles are represented in the bottom figures.

Figure 4. Energy per cycle (top) and power (bottom) extracted as a function of the current applied for the three kinds of cycles shown in Fig. 3.

Figure 5. OCV measurements for individual carbon electrodes coated with cationic (PDADMAC) and anionic (PSS) polymers. AC and AB represent the respective voltage rise.

Figure 6. OCV measurements for single electrodes coated with: two different anionic polymers, PSS (a) and PAA (b), and the cationic polymer PDADMAC (c). The dashed line in a) shows the response of $60 \mathrm{mM}$ PSS with lower molecular weight $\left(\mathrm{M}_{w} \approx 70000\right)$. The dashed line in $\left.\mathrm{c}\right)$ corresponds to $60 \mathrm{mM}$ $\mathrm{M}_{w} \approx 200$ 000-350 000 PDADMAC.

Figure 7. a) Electrophoretic mobility as a function of $\mathrm{NaCl}$ concentration.

b) Modulus (top) and phase (bottom) of the dynamic electrophoretic mobility versus frequency. Both measurements for non-treated carbon particles (Norit) and for same particles coated with PSS and PDADMAC. 
Figure 8. OCV kinetics for polymer-treated graphite films (dashed lines) and carbon films (solid lines). 
List of Figures

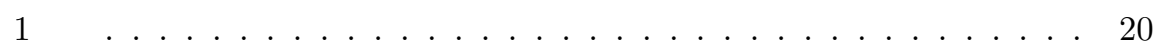

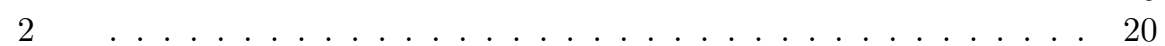

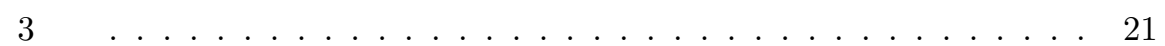

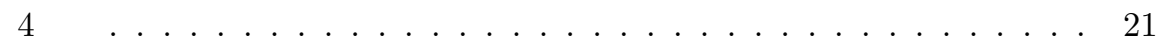

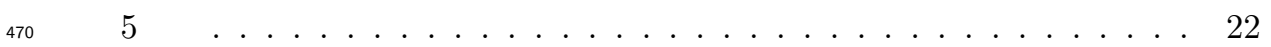

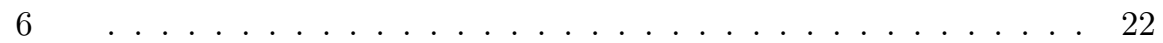

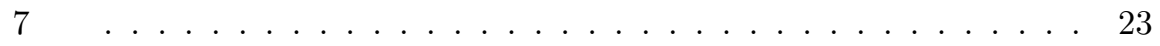

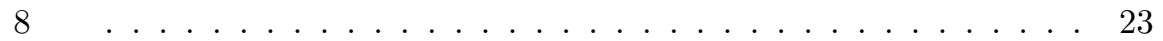




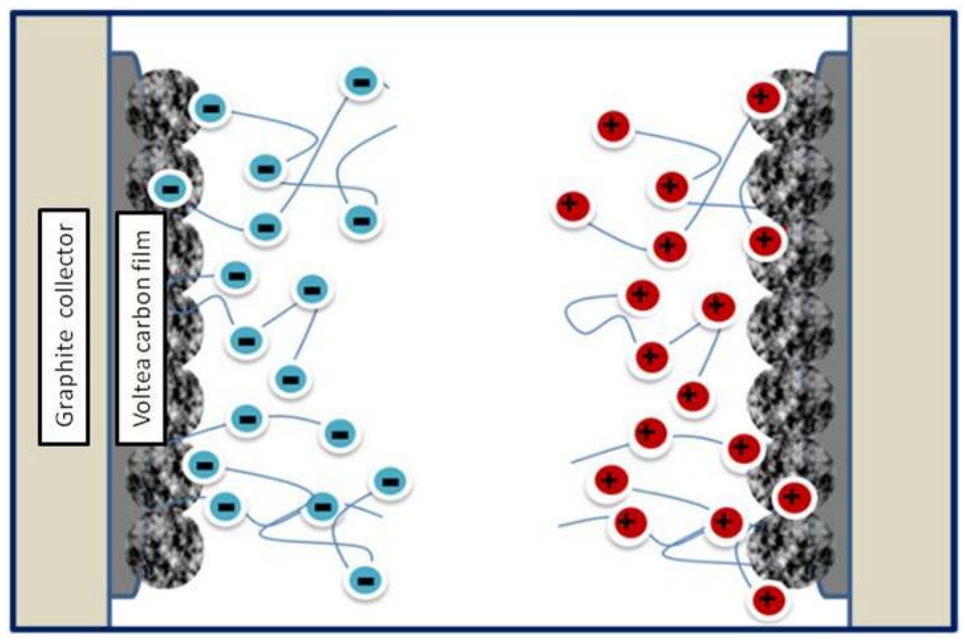

Figure 1

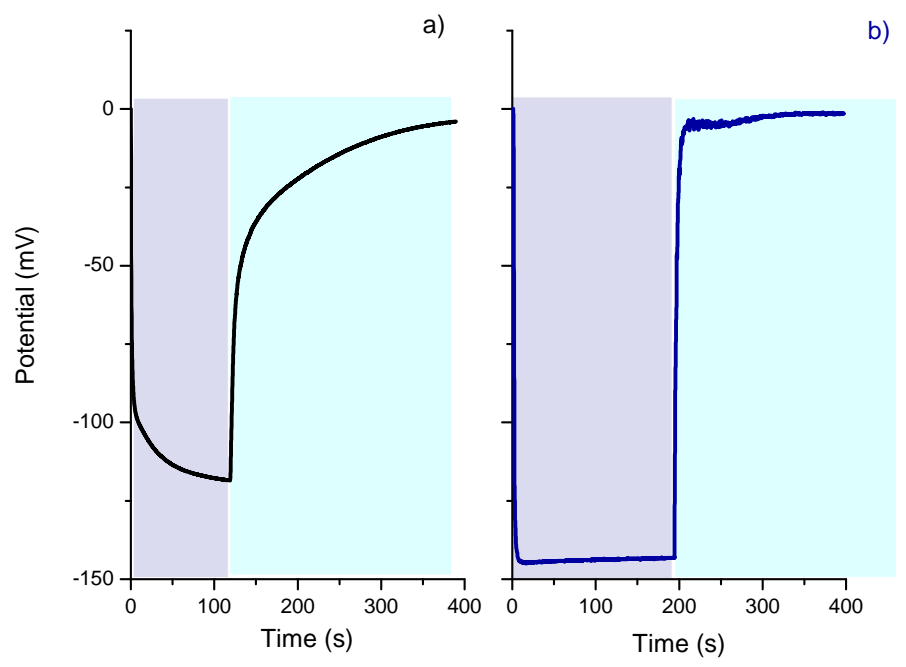

Figure 2 


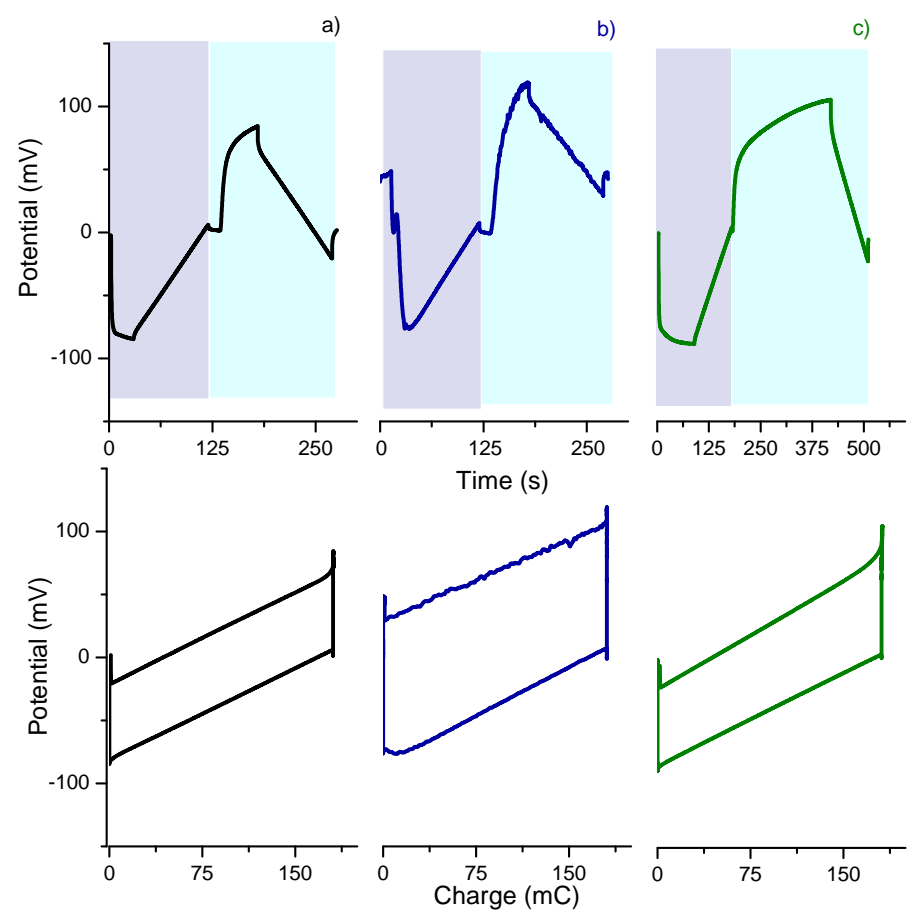

Figure 3

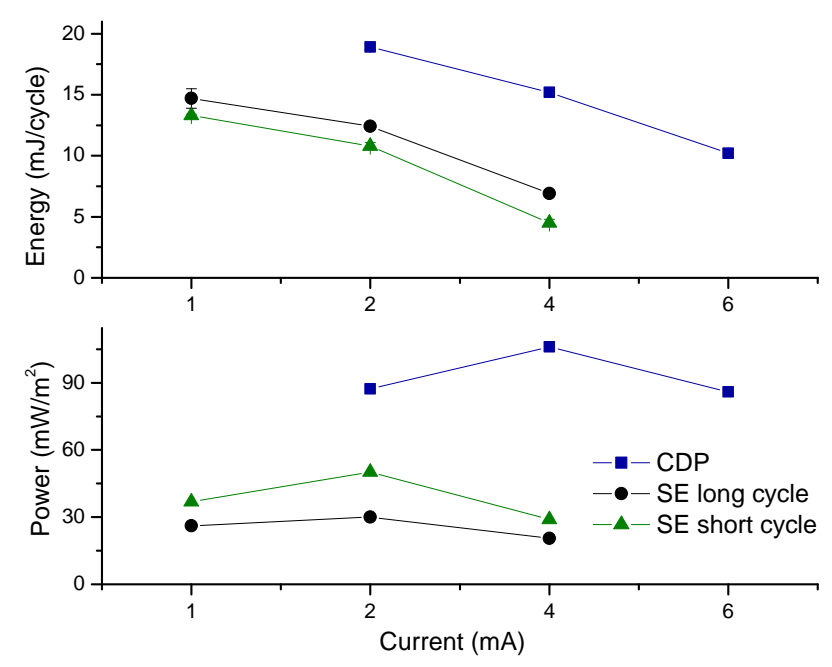

Figure 4 


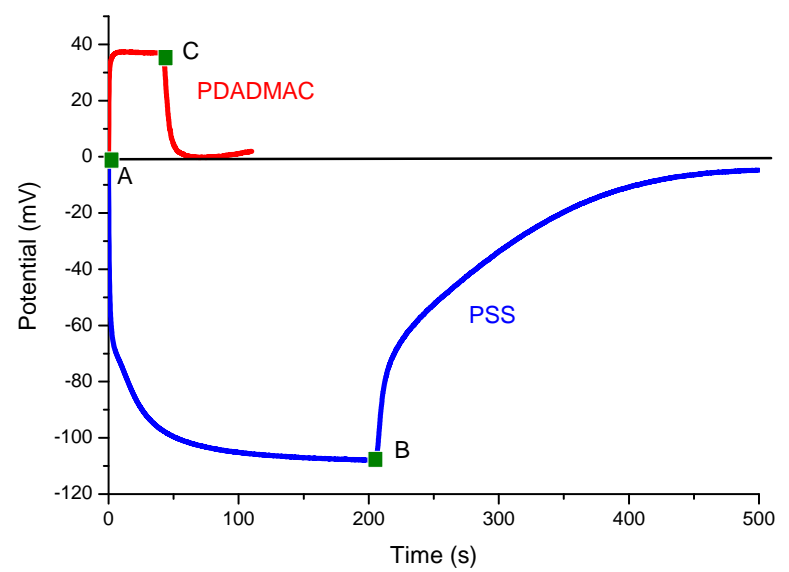

Figure 5

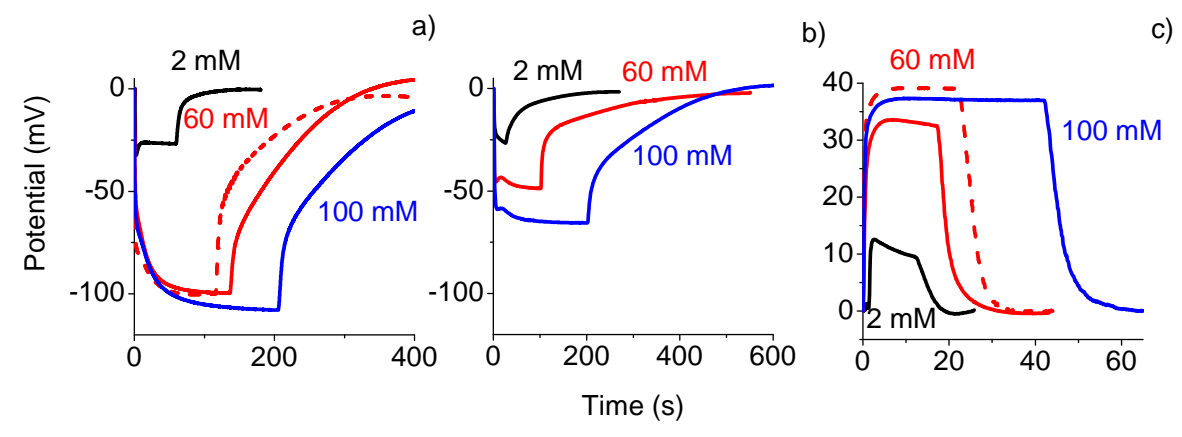

Figure 6 
a)

b)
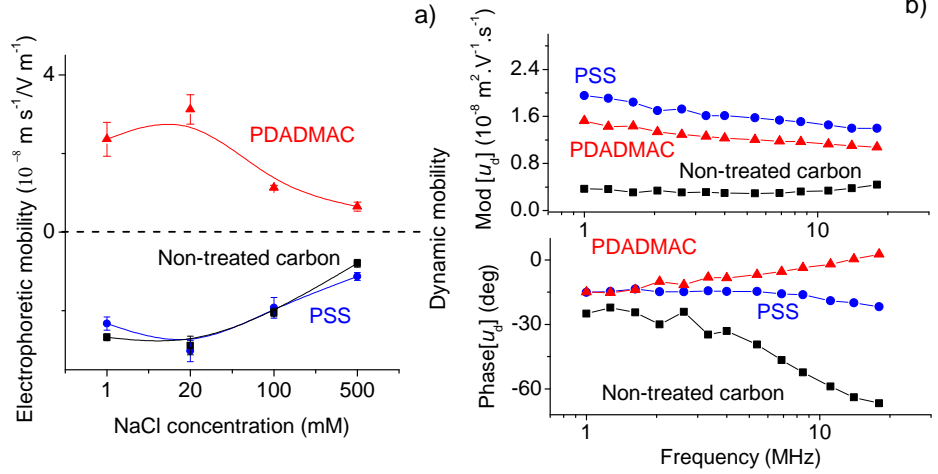

Figure 7

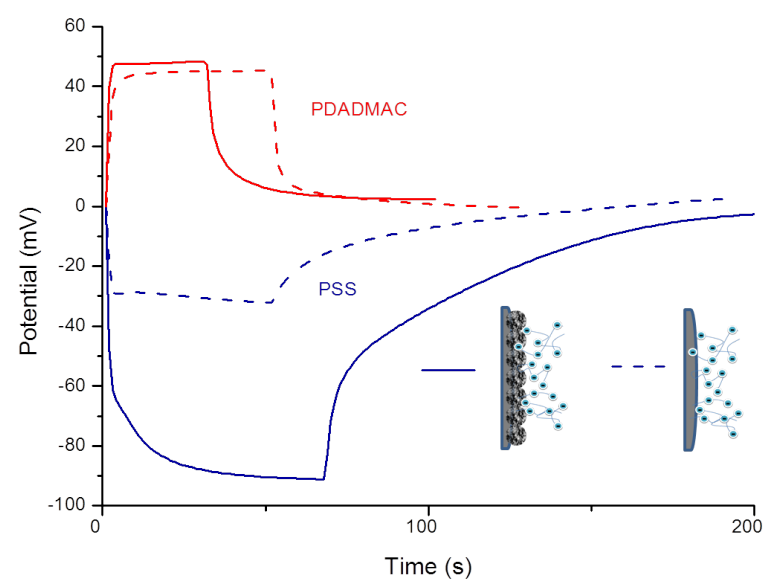

Figure 8 